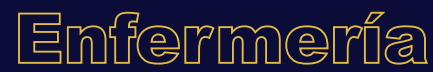

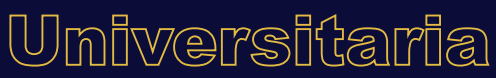

\section{Teoría de Kolcaba: propuesta para el cuidado de niños y niñas chilenos enfermos de cáncer}

\author{
Kolcaba Theory: A proposal to caring \\ Chilean children with cancer
}

\section{Teoria de Kolcaba: proposta para o cuidado de crianças chilenas doentes de câncer}

\author{
C.A. Delgado-Riffo ${ }^{{ }^{*}}$, S. Valenzuela-Suazo ${ }^{b}$ \\ ORCID \\ ${ }^{\mathrm{a}} 0000-0002-6891-0663$ \\ bo000-0002-1308-4835 \\ ${ }^{1}$ Universidad de Concepción, Facultad de Enfermería, Concepción, Chile \\ Recibido: 14 enero 2020 \\ Aceptado: 27 septiembre 2020
}

RESUMEN

Introducción: La comodidad o bienestar es un concepto que ha estado presente como objetivo del cuidado de enfermería desde sus inicios como disciplina y ha demostrado ser un factor que mejora las condiciones de salud de las personas. Enfermería tiene la responsabilidad de entregar el mejor cuidado posible, fundamentando su quehacer en el conocimiento científico humanista y en el uso de teorías que emergen desde la disciplina. Dentro de éstas destacan por su aplicabilidad las teorías de rango medio, una de ellas es la teoría de la comodidad de Kolcaba, que ha sido utilizada en diversos contextos de salud. Por consiguiente, el presente artículo tiene como objetivo entregar una propuesta de aplicación de la teoría de la comodidad de Kolcaba para el cuidado de niños y niñas enfermos/as de cáncer en unidades pediátricas chilenas.

Desarrollo: Se realizó un análisis de los constructos que conforman la teoría, articulándolos con evidencias originadas desde su aplicación e integrándolos con la realidad que les toca enfrentar a los pacientes, sus familias y los equipos de salud oncológicos infantiles que también han sido documentadas.

*Autora para correspondencia. Correo electrónico: claudiandreadr@gmail.com https://doi.org/10.22201/eneo.23958421e.2020.4.788

1665-7063/@ 2020 Universidad Nacional Autónoma de México, Escuela Nacional de Enfermería y Obstetricia. Este es un artículo Open Access bajo la licencia CC BY-NC-ND (http://creativecommons.org/licenses/by-nc-nd/4.o/). 
Conclusión: Se presenta una propuesta de aplicación de la teoría de la comodidad en pacientes oncológicos pediátricos. De igual forma, se reconoce que esta teoría permite entregar un cuidado holístico al paciente y su familia.

Palabras claves: Comodidad del paciente; niño; oncología; teoría de enfermería; enfermería oncológica; Chile.

\section{ABSTRACT}

Introduction: Wellbeing is an objective which has been present in nursing care since the beginnings of the discipline. Nursing has the responsibility of delivering the best possible care, based on humanistic scientific knowledge and the use of theories emerging from the discipline itself. Among these, the medium range theories can be highlighted because of their application potential. One of these is the Theory of Kolcaba, which has been utilized in diverse health related contexts. The following article has the objective of presenting a proposal on the application of the Theory of Kolcaba to address the care of children with cancer in pediatric units in Chile.

Development: An analysis was carried out on the constructs structuring the Kolcaba Theory, articulating them with evidence and integrating them into the context of pediatric patients with cancer and their families, and the health teams.

Conclusion: A proposal of application of the Theory of Kolcaba on pediatric patients with cancer is presented, acknowledging that this theory allows the delivery of holistic care to these patients and their families.

Keywords: Patient comfort; child; oncology; nursing theory; oncology nursing; Chile.

\section{RESUMO}

Introdução: O conforto ou bem-estar é um conceito que está presente como objetivo do cuidado de enfermagem desde seu início como disciplina e tem se mostrado um fator que melhoria as condições de saúde das pessoas. A enfermagem tem a responsabilidade de prestar o melhor cuidado possivel, fundamentando seu afazer no conhecimento científico humanístico e no uso de teorias que emergem da disciplina. Dentre elas salientam pela sua aplicabilidade as teorias de médio alcance, uma delas é a teoria do conforto de Kolcaba, que tem sido utilizada em diversos contextos de saúde. Por consequência, o presente artigo tem como objetivo apresentar uma proposta de aplicação da teoria do conforto de Kolcaba para o cuidado de crianças doentes de câncer em unidades pediátricas chilenas.

Desenvolvimento: Foi realizada uma análise dos constructos que conformam a teoria, articulando-os às evidências originadas da sua aplicação e integrando-os à realidade que têm enfrentado os pacientes, suas famílias e as equipes de saúde oncológicas infantis e que também têm sido documentadas.

Conclusão: Apresenta-se uma proposta de aplicação da teoria do conforto em pacientes oncológicos pediátricos. Da mesma forma, reconhece-se que esta teoria permite prestar um cuidado holístico ao paciente e sua família.

Palavras chave: Conforto do paciente; criança; oncologia; teoria da enfermagem; enfermagem oncológica; Chile. 


\section{INTRODUCCIÓN}

El presente artículo tiene como objetivo entregar una propuesta de aplicación de la teoría de la comodidad de Kolcaba para el cuidado de niños y niñas chilenos enfermos de cáncer atendidos en unidades pediátricas. Se inicia por la definición de sus principales conceptos, integrándolos con publicaciones que den cuenta de la problemática a la que se enfrentan los pacientes, sus familias y los equipos de salud de oncología infantil.

La comodidad es un efecto muy esperado y buscado por los pacientes y su familia, además de constituir un propósito dentro de los cuidados de enfermería. Se estima que, a pesar de que las enfermeras y enfermeros saben de manera intuitiva qué es la comodidad y de qué manera favorecerla en sus pacientes, el foco de la tarea continúa siendo enfrentar la patología y se trabaja con el fin de no generar incomodidad, lo que entrega una sensación neutra de comodidad más que de buscarla activamente, como la ausencia de un síntoma específico².

A partir del cambio epidemiológico ocurrido en Chile, una de las patologías más prevalentes es el cáncer. Actualmente es la segunda causa de muerte en la población infantil mayor de cinco años desde la década de 1980, situación que se repite en Europa y Estados Unidos². En Chile, la incidencia de cáncer infantil en el año 2014 correspondió a 16 por 100 ooo niños/as³. Los tres cánceres más frecuentes en la población chilena menor de 15 años son las leucemias (35\%), los tumores del Sistema Nervioso Central (17\%) y los linfomas (10\%)4.

En consecuencia, el Ministerio de Salud de Chile (MINSAL) creó en 1988 el Programa Infantil Nacional de Drogas Antineoplásicas (PINDA), que se encuentra inserto en el Programa Nacional de Cáncer. Dicho programa tiene como población beneficiaria a todas y todos los menores de 15 años con sospecha o confirmación de enfermedad oncológica y aquellas(os) con recaída de cáncer infantil hasta los 24 años. Además, los cánceres infantiles fueron incorporados dentro de las primeras 25 patologías del Sistema de Garantías Explícitas en Salud (GES), según el Decreto $\mathrm{N}^{\circ} 170$ del año $2005^{3}$. Cabe destacar que, a cinco años de ser atendidos en el sistema público de salud, los pacientes han alcanzado $71.4 \%$ de sobrevida ${ }^{2,3,5,6}$.

Por otra parte, el tratamiento de los niños y niñas con esta enfermedad incorpora procedimientos altamente complejos y agresivos, por tanto, es imprescindible que el equipo de salud responsable de la atención de estos pacientes cuente con un alto grado de capacitación para entregar una atención de alta calidad que permita evitar y/o detectar precozmente complicaciones innecesarias5.

Como parte de este equipo oncológico, el profesional de enfermería asume la responsabilidad de entregar el mejor cuidado posible. De acuerdo con el Código Sanitario chileno, desde 1997 esto se encuentra estipulado legalmente en el siguiente mandato que reconoce al cuidado como eje central del quehacer de enfermería pues: "Los servicios profesionales de la enfermera comprenden la gestión del cuidado en lo relativo a promoción, mantención y restauración de la salud, la prevención de enfermedades o lesiones y la ejecución de acciones derivadas del diagnóstico y tratamiento médico y el deber de velar por la mejor administración de los recursos de asistencia para el paciente"?

Por consiguiente, esto hace necesario sustentar el quehacer de enfermería en el conocimiento específico de la disciplina, por medio del uso de sus teorías, siendo este uno de sus mayores desafíos ${ }^{8}$ debido a la escasa introducción de éstas en la programación del cuidado por parte de enfermeras y enfermeros a cargo de la atención directa de pacientes. Se reconoce que estos profesionales utilizan constantemente matices de esas teorías como parte de sus labores asistenciales aún sin reconocerlo, lo cual reafirma la necesidad de su aplicación al brindar atención a las personas y comunidades que se encuentran bajo el cuidado de enfermería?. 
Durante la década de 1990 emergieron las teorías de rango medio, las cuales fundamentan el cuidado de enfermería y se caracterizan por poseer particularidades sencillas y puntuales, haciéndolas más cercanas alquehacer asistencialdebido a sus características que permiten centrarse en un determinado fenómeno muy concreto de la práctica de enfermería gracias a su nivel menor de abstracción ${ }^{10}$. De esta forma, orientan de manera más clara la práctica y favorecen la creación de nuevas preguntas de investigación que, al ser respondidas, aportan mayor conocimiento a la teoría en el espacio donde puede focalizarse.

Dentro de estas teorías de rango medio, la teoría de la comodidad de Kolcaba ha sido usada en diversos contextos de cuidado como personas hospitalizadas con cuadros depresivos ${ }^{11}$, en perianestesia ${ }^{12}$, en pacientes oncológicos ${ }^{1,13,14}$ y sus cuidadores ${ }^{15,16}$, en adultos mayores ${ }^{17}$, entre otros.

Los niños y niñas enfermos de cáncer demandan un cuidado que constantemente desafía a enfermería, producto del desgaste emocional que ocasiona, debido a que vivir con una enfermedad potencialmente mortal influye sobre diversas dimensiones de la vida del paciente y su familia. Las patologías oncológicas son algunas de las que originan más dolor, sufrimiento, ansiedad, estrés y miedo a la muerte y/o progresión o recurrencia de la enfermedad en el paciente, la familia y el equipo a cargo del cuidado, formándose un vínculo entre ellos/as como resultado de la cercanía que se produce durante la rutina asistencial ${ }^{18}$.

\section{DESARROLLO}

\section{a) Contexto teórico}

Katharine Kolcaba publicó en 1991 su concepto de comodidad, elaboró un esquema que agrupaba los aspectos que lo componen y utilizó este concepto como objetivo del cuidado' ${ }^{19}$. El o los conceptos son ideas que componen elementos para formación de teorías determinadas, en el caso de la teoría de la comodidad, estos conceptos son limitados en su cantidad y poseen un bajo nivel de abstracción, lo que hace fácil su definición y medición ${ }^{12}$.

Kolcaba define comodidad para el cuidado de la salud como "el estado inmediato de ser fortalecido por tener las necesidades de alivio, tranquilidad y trascendencia abordadas en los cuatro contextos de la experiencia humana holística: física, psicoespiritual, sociocultural y ambiental”2o.

El contexto físico se refiere a las sensaciones corporales, el contexto psicoespiritual representa a la conciencia interna del yo, pudiendo contener la relación con un ser superior. El contexto sociocultural corresponde a las relaciones interpersonales, sociales y familiares y el contexto ambiental incluye infraestructura, temperatura, iluminación, etcétera ${ }^{20}$. Al relacionar los tres estados de comodidad con los cuatro contextos de la experiencia de comodidad se produce una red de 12 celdas llamada estructura taxonómica (Tabla 1), la cual ha sido usada en el área de investigación y práctica clínica en diversos contextos de cuidado².

Por otro lado, la teoría de la comodidad afirma que una intervención reconfortante, dada en el tiempo, aumenta la comodidad de los enfermos en situaciones de atención de salud estresantes ${ }^{12}$. Las necesidades de cuidado de la salud emergen desde este tipo de situaciones insertas en el cuidado de la salud en los sistemas tradicionales de apoyo, donde las necesidades son identificadas, pero no pueden ser satisfechas por el paciente/familia ${ }^{19,20}$. Estas últimas pueden ser físicas, psicoespirituales, sociales y ambientales y se manifiestan por medio del seguimiento, informes verbales y no verbales, parámetros fisiopatológicos, educación, apoyo, asesoramiento e intervención financiera. 
Tabla 1. Estructura taxonómica de la comodidad según Kolcaba

\begin{tabular}{|c|c|c|c|}
\hline \multirow[t]{2}{*}{ Contexto } & \multicolumn{3}{|c|}{ Tipos de comodidad } \\
\hline & Alivio & Tranquilidad & Trascendencia \\
\hline \multicolumn{4}{|l|}{ Físico } \\
\hline \multicolumn{4}{|c|}{ Psicoespiritual } \\
\hline \multicolumn{4}{|l|}{ Ambiental } \\
\hline Social & & & \\
\hline
\end{tabular}

Fuente: Dowd T. La teoría del confort. En: Raile-Alligood M. Modelos y teorías en enfermería. $8^{\circ}$ ed. Barcelona, España: Elsevier; $2015^{21}$.

Una preocupación constante para la familia y el equipo a cargo de niños y niñas enfermos/as de cáncer es evitar y/o manejar el dolor; síntoma considerado como una experiencia subjetiva que debe ser idealmente autodeclarado, lo cual no siempre es posible en los/as niños/as por diferentes motivos como la edad, capacidad cognitiva o verbal, entre otras ${ }^{22}$. Por lo tanto, es una necesidad constante su valoración por medio de instrumentos validados y la comunicación persistente y fluida por parte de profesionales de enfermería y la familia. Asimismo, la hospitalización en las/os niñas/os constituye un contexto que acrecienta su propia fragilidad, debido a que se encuentran sometidos a estímulos estresantes y seguramente amenazantes para su integridad física ${ }^{22}$.

Las variables intervinientes corresponden a factores que median en la percepción de comodidad. Pueden ser experiencias pasadas, edad, actitud, estado emocional, sistemas de apoyo, el pronóstico, situación financiera u otras ${ }^{19,20}$, además de que suelen ser estáticas y el equipo de salud tiene poco control sobre ellas. En el caso de los pacientes oncológicos pediátricos, las hospitalizaciones reiteradas y frecuentes a las que se someten durante su tratamiento son eventos que generan ansiedad de manera repetida. Por su parte, los cuidadores, generalmente los padres, presentan alto grado de estrés, angustia y miedo, que pueden desencadenar crisis de ansiedad ${ }^{23}$. Estas situaciones afectarán el grado de comodidad percibido por los pacientes y sus familias.

Las intervenciones de comodidad han sido definidas como "acciones concretas de las enfermeras que producen un estado inmediato de comodidad en el receptor" ${ }^{\prime \prime}$. Kolcaba agrega que cuando son administradas en forma continua, se produce una tendencia hacia mayores niveles de comodidad en el tiempo. Estas intervenciones pueden ser fisiológicas, sociales, económicas, psicológicas, espirituales, ambientales y físicas ${ }^{19,20}$. Un ejemplo de esto es la gestión de apoyo con la Risoterapia. La incorporación de payasos en el hospital, quienes ofrecen humor, juego, distracción y creatividad, se ha convertido en una estrategia que contribuye a la experiencia positiva de hospitalización por parte de niñas y niños internados, ya que se relaciona con emociones positivas y bienestar. Además, se demostró que al estar presentes durante la realización de procedimientos dolorosos como punción lumbar o aspiración de médula ósea los ayuda a controlar el miedo²4.

Otro concepto de esta teoría son los comportamientos de búsqueda de la salud, es decir, las acciones realizadas por el paciente/familia con el objetivo de conseguir la salud pudiendo ser internas, externas o incluso la búsqueda de una muerte pacífica, acciones que a su vez son consultadas con la/el enfermera/ $\mathrm{o}^{19,20}$. Es aquí donde los cuidados posteriores a la administración del tratamiento oncológico entregados por los padres de los pacientes cobran importancia, por consiguiente, es responsabilidad del personal de enfermería educar, despejar dudas, disminuir temores y supervisar el cumplimiento de las indicaciones, procurar que la familia vea alterada su rutina lo menos posible y evitar al máximo las complicaciones. 
Kolcaba define la integridad institucional como "los valores, la estabilidad financiera y la integridad de las organizaciones de atención de salud a nivel local, regional, estatal y nacional”"17. Se entiende como instituciones no exclusivamente al sistema hospitalario, sino además a las corporaciones o agrupaciones de diferente origen que proveen cuidado a personas con diferentes estados de salud, como empresas de hospitalización domiciliaria, hogares de ancianos, etc., las cuales poseen características que favorecen prácticas y políticas que facilitan la comodidad ${ }^{19,20}$. La autora da ejemplos concretos de variables relacionadas con esta definición como satisfacción usuaria, reducción de costos, mejora en el acceso, disminución de las tasas de morbilidad, de las hospitalizaciones y reingresos, entre otras ${ }^{25}$.

En el caso de Chile, la Superintendencia de Salud del MINSAL establece claramente cuáles son las garantías de oportunidad exigibles por las personas menores de 15 años enfermas de cáncer. Por ejemplo, para el caso de leucemias y tumores sólidos, establecer el diagnóstico, incluida la etapa de éste dentro de los 14 y 37 días respectivamente desde la sospecha; inicio de quimioterapia dentro de las 24 horas desde el diagnóstico, primer control de seguimiento al término del tratamiento a los 7 días para el caso de leucemia y 30 días en linfomas y tumores sólidos²6.

Las políticas óptimas se refieren a los protocolos y procedimientos desarrollados a nivel local o regional con la mejor evidencia disponible, para guiar el quehacer del equipo de salud, así como favorecer el acceso y la asistencia ${ }^{19,20}$. En Chile, el MINSAL cuenta con las guías clínicas AUGE para el manejo de las leucemias, los linfomas y tumores sólidos en menores de 15 años, las cuales permiten el manejo estandarizado de los cánceres infantiles ${ }^{27}$.

Por su parte, el uso de estos protocolos y procedimientos en un grupo específico de pacientes o en el paciente/familia es lo que Kolcaba define como prácticas recomendables ${ }^{19,20}$. A partir del año 2002 cuando el cáncer infantil fue incorporado dentro de las tres primeras patologías al listado de patologías piloto AUGE, las personas menores de 15 años desde la etapa de sospecha de cáncer tienen asegurado el acceso, la oportunidad de atención y protección financiera².

Sin embargo, desde el año 1988 se están realizando esfuerzos para estandarizar el tratamiento del cáncer en la población infantil, cuando la Comisión Nacional del Cáncer del MINSAL implementó el Programa Nacional de Drogas Antineoplásicas (PINDA) para niños y niñas menores de 15 años enfermos/as de cáncer con el fin de alcanzar la curación del mayor número de pacientes, con la mejor calidad de vida . Comenzó con 13 protocolos que habían demostrado su utilidad (se excluyen las ramas experimentales), se trabajó en conjunto con grupos colaborativos internacionales como el grupo alemán Berlin-Frankfurt-Muenster (BFM) para leucemias y linfomas, los grupos americanos Wilms Tumour Study e Intergroup Rhabdomyosarcoma Study Group para los tumores respectivos, entre otros 5 . Estos hitos demuestran la preocupación del Estado chileno por establecer políticas para este grupo de personas, lo cual es necesario para que las/os pacientes alcancen mayores niveles de comodidad, de acuerdo con lo expuesto por la teoría de Kolcaba.

Kolcaba y Dimarco expresan que el uso de la teoría de la comodidad en niñas y niños entrega a la enfermería pediátrica razones para mejorar la comodidad de estos y la de sus familias ${ }^{28}$. Además, entregan siete proposiciones a considerar por enfermería en el cuidado de niños y niñas:

1. Las y los niñas/os y sus familias tienen respuestas amplias a estímulos complejos.

2. El confort es un resultado deseable, positivo y holístico que es adecuado para la enfermería pediátrica.

3. Las/os niñas/os y sus familias se esfuerzan activamente por satisfacer sus necesidades básicas de bienestar, necesitando en ciertas ocasiones de la ayuda de enfermería. 
4. Las/os niñas/os y sus familias varían significativamente en su necesidad para ciertos niveles de comodidad.

5. Es más fácil prevenir las molestias (incomodidad) fisiológicas y/o psicológicas, siendo mejor para los pacientes y sus familias.

6.Cuando las molestias de origen ambiental y/o fisiológico no pueden impedirse, los niños y sus familias pueden ser ayudados para experimentar una trascendencia parcial o completa a través de las intervenciones de confort que transmiten esperanza, éxito, cuidado y apoyo para su miedo.

7. Cuando las enfermeras aplican la teoría de la comodidad, reconocen la particularidad y complejidad de cada niña/o dentro del contexto del sistema familiar.

La autora de la teoría declara que el conocimiento de ésta permite a enfermeras y enfermeros guiar sus intervenciones en los contextos físicos, psicoespirituales, sociales y ambientales ${ }^{25}$. De esta forma, posibilita ajustar un plan de cuidados que contenga las intervenciones que funcionan para cada paciente y comunicarlas al equipo a cargo de la atención ${ }^{28}$.

\section{b) Propuesta para el cuidado de niñas y niños enfermas/os de cáncer}

Al aplicar la matriz a las percepciones vivenciadas más frecuentemente por los(as) niños y niñas que sufren de cáncer, es posible construir una propuesta de necesidades específicas para este grupo de pacientes, basadas en la estructura taxonómica propuesta por Kolcaba, la cual se establece desde la experiencia profesional que una de las autoras posee con este grupo de niñas y niños (Tabla 2).

\begin{tabular}{|c|c|c|c|}
\hline $\begin{array}{l}\text { Contexto/ } \\
\text { Acciones }\end{array}$ & Alivio & Tranquilidad & Trascendencia \\
\hline \multirow[t]{2}{*}{ Físico } & $\begin{array}{l}\text { Necesidad de manejo } \\
\text { del temor frente al } \\
\text { dolor producido por } \\
\text { procedimientos y/o efectos } \\
\text { secundarios del tratamiento }\end{array}$ & $\begin{array}{l}\text { Necesidad de salas y unidad } \\
\text { del paciente cómodas }\end{array}$ & $\begin{array}{l}\text { Necesidad de educación y } \\
\text { apoyo frente a los cambios } \\
\text { en la imagen corporal } \\
\text { secundarias al tratamiento } \\
\text { oncológico }\end{array}$ \\
\hline & $\begin{array}{l}\text { Necesidad de manejo del } \\
\text { temor a cambios en la } \\
\text { imagen corporal }\end{array}$ & $\begin{array}{l}\text { Necesidad de descanso, de } \\
\text { silencio }\end{array}$ & $\begin{array}{l}\text { Necesidad de educación } \\
\text { en prevención de efectos } \\
\text { secundarios al tratamiento }\end{array}$ \\
\hline \multirow[t]{2}{*}{ Psicoespiritual } & $\begin{array}{l}\text { Necesidad de manejo de la } \\
\text { tristeza por separación de la } \\
\text { familia }\end{array}$ & $\begin{array}{l}\text { Necesidad de apoyo } \\
\text { psicológico para disminuir } \\
\text { ansiedad producida por el } \\
\text { diagnóstico y tratamiento }\end{array}$ & $\begin{array}{l}\text { Comunicación fluida con el } \\
\text { equipo de enfermeras }\end{array}$ \\
\hline & $\begin{array}{l}\text { Necesidad de manejo del } \\
\text { temor por encontrarse en } \\
\text { un ambiente extraño y hostil }\end{array}$ & $\begin{array}{l}\text { Necesidad de } \\
\text { acompañamiento espiritual }\end{array}$ & $\begin{array}{l}\text { Necesidad de sentirse } \\
\text { acompañado/a por familia y } \\
\text { amigos/as }\end{array}$ \\
\hline Ambiental & $\begin{array}{l}\text { Necesidad de privacidad por } \\
\text { salas compartidas }\end{array}$ & $\begin{array}{l}\text { Necesidad de descanso, } \\
\text { de silencio, de horarios } \\
\text { establecidos para recreación } \\
\text { con TV }\end{array}$ & $\begin{array}{l}\text { Mantener la privacidad } \\
\text { de acuerdo con la edad } \\
\text { al momento del aseo } \\
\text { personal, cambio de ropa o } \\
\text { satisfacción de la necesidad } \\
\text { de eliminación }\end{array}$ \\
\hline
\end{tabular}




\begin{tabular}{ll}
$\begin{array}{l}\text { Necesidad de mantener una } \\
\text { iluminación y temperatura } \\
\text { de la sala de hospitalización } \\
\text { adecuada que favorezca el } \\
\text { descanso }\end{array}$ & $\begin{array}{l}\text { Necesidad de mantener el } \\
\text { autocuidado de acuerdo } \\
\text { con la edad y capacidad } \\
\text { cognitiva }\end{array}$ \\
$\begin{array}{l}\text { Necesidad de recibir } \\
\text { información del equipo } \\
\text { de salud acerca de su } \\
\text { condición, tratamiento y } \\
\text { procedimientos, de acuerdo } \\
\text { a la edad }\end{array}$ & $\begin{array}{l}\text { Necesidad de minimizar el } \\
\text { riesgo de deserción escolar }\end{array}$ \\
$\begin{array}{l}\text { Necesidad de evitar el } \\
\text { uso de lenguaje técnico } \\
\text { del equipo que dificulta } \\
\text { comprensión y participación } \\
\text { en el proceso de tratamiento }\end{array}$ & $\begin{array}{l}\text { el estancamiento en el } \\
\text { proyecto de vida }\end{array}$ \\
\hline
\end{tabular}

Con base en lo anterior, es posible observar que una vez distinguidas las necesidades según el contexto de pertenencia, la delimitación por tipo de acción o gestión a realizar por personal de enfermería para favorecer la comodidad, permite concretar con claridad los propósitos de las intervenciones posibles.

Todas las necesidades en los cuatro contextos propuestos son fuertemente alteradas desde el momento del diagnóstico, tanto para quien sufre la enfermedad, como para su entorno más intimo. La estructura hospitalaria, sus rutinas, horarios, entre otras restricciones, pueden impactar fuertemente sobre la comodidad del paciente y familia, quienes podrían incluso, verlos como una amenaza.

Las dificultades para el profesional de enfermería de lograr una comunicación fluida con el niño o niña, imprescindible para obtener información acerca de las necesidades y estado del paciente, son ocasionadas por la etapa del desarrollo y hacen aún más desafiante el trabajo con este grupo, pues exigen a la enfermera el despliegue de habilidades que le permitan obtener información válida basada en el lenguaje no verbal del paciente. En este punto, los padres son un pilar fundamental, en la medida en que pueden interpretar las necesidades del menor.

Asimismo, la gran carga emocional que posee el diagnóstico de cáncer en los padres, la incertidumbre sobre el pronóstico, los riesgos del tratamiento y los cuidados necesarios en el domicilio, hacen imprescindible considerar y hacer partícipes a los padres al momento de la valoración de comodidad, ya que "cuando un niño o niña se enferma de cáncer, toda la familia se enferma de cáncer". Debido a esto, las autoras consideran que el uso de la matriz propuesta en la teoría de la comodidad permitiría revelar las necesidades del paciente y su familia, además favorecería una adecuada priorización de éstas, lo que apoyaría la elaboración de un plan de cuidados individualizado para cada paciente, posibilitaría establecer metas y su posterior evaluación. De esta forma, su implementación en unidades oncológicas pediátricas mejoraría la forma en que es entregado el cuidado, de forma que sea individual, integral, seguro y amoroso.

\section{CONCLUSIÓN}

Se concluye que el uso de un marco teórico propio en el quehacer asistencial de enfermería, como las teorías de mediano rango, aporta a enfermeras y enfermeros herramientas propias para entregar 
el mejor cuidado posible, debido a que ofrecen un camino de acción preciso que da respuesta a cuestiones concretas de la práctica.

Aunado a lo anterior, la teoría de la comodidad de Kolcaba permite brindar un cuidado holístico al paciente y familia, más aún cuando se ven afectadas tantas necesidades en niños y niñas con patologías oncológicas, ya que considera factores internos y externos que influyen en el estado de comodidad en diferentes contextos de la experiencia de la persona, aumentando de esta forma el grado de satisfacción de los y las usuarios/as.

Finalmente, esta teoría de rango medio ha sido utilizada en diversos contextos, como pacientes con cáncer y sus cuidadores y pacientes pediátricos, entre otros, por lo que su uso es posible en niños y niñas enfermos de cáncer y sus familias, lo que ha sido incentivado por Kolcaba, quien entrega proposiciones a las enfermeras y enfermeros a cargo del cuidado de menores y sus familias.

\section{RESPONSABILIDADES ÉTICAS}

Protección de personas y animales. Las autoras declaran no haber realizado experimentos en seres humanos ni en animales para el desarrollo de esta investigación.

Financiamiento. Ninguno.

Conflicto de intereses. Las autoras declaran no tener conflicto de intereses.

\section{REFERENCIAS}

1. Kim KS. Kwon SH. Comfort and quality of life of cancer patients. Asian Nurs Res. 2007; 1(2): 125-35. https://doi.org/10.1016/S1976-1317(08)60015-8

2. Ministerio de Salud. Guía Clínica Leucemia en personas menores de 15 años. Santiago, Chile; MINSAL; 2010. https://bit.ly/3kAUYsL

3. Ministerio de Salud. Plan nacional de cáncer 2018-2028. Chile: MINSAL; 2019. https://bit.ly/zbRIetH

4. Ministerio de Salud. Cáncer infantil: Chile trabaja para alcanzar un 80\% de sobrevida al año 2020 . Santiago, Chile: MINSAL; 2016. https://bit.ly/zoozuIg

5. Ministerio de Salud. Historia de PINDA. Santiago: PINDA Chile; 2010. https://bit.ly/ze3R3Uc

6. El estetoscopio. Programa PINDA y cáncer infantil. En: Sociedad chilena de pediatría. Programa PINDA y cáncer infantil. 30 años de labor fundamental. El estetoscopio. 2017; 15(93): 5-10. https://bit.ly/301v40u

7. Ministerio de Salud Pública. Código Sanitario. Libro V Del ejercicio de la Medicina y profesiones afines. Chile: MINSAL; 2011. https://bit.ly/2PioqYO

8. Rodríguez-Campo VA, Valenzuela-Suazo S. Teoría de los cuidados de Swanson y sus fundamentos, una teoría de mediano rango para la enfermería profesional en Chile. Enfermería glob. 2012;11(4): 316-22. https://doi.org/10.6018/eglobal.11.4.141391

9. Agama-Sarabia A, Cruz-Rojas L, Ostiguín-Meléndez R. Teoría: Alcances y niveles para explicar la práctica de enfermería. Biblioteca Las Casas. 2011; 7(3): 1-11. https://bit.ly/zeziBsQ

10. Durán-de Villalobos MM. La teoría, soporte de la ciencia y práctica de enfermería: tendencias. Av. enferm. 2012; 30(1): 9-12. Disponible en: https://bit.ly/381wTX5

11. Alves-Apóstolo JL. Kolcaba K. The effects of guided imagery on comfort, depression, anxiety, and stress of psychiatric inpatients with depressive disorders. Arch Psychiatr Nurs. 2009; 23(6):403-11. https://doi.org/10.1016/j.apnu.2008.12.003 
12. Wilson L, Kolcaba K. Practical application of comfort theory in the perianesthesia setting.J Perianesth Nurs. 2004; 19(3): 164-73. https://doi.org/10.1016/j.jopan.2004.03.006

13. Tuncer G, Yucel S. Comfort and anxiety levels of women with early stage breast cancer who receive radiotherapy. Asian Pac J Cancer. 2014; 15(5): 2109-14.

https://doi.org/10.7314/apjcp.2014.15.5.2109

14. Carvalho-Alves Soares ML, Soares-Figueiredo Trezza MC, Barros-de Oliveira SM, Cavalcante-de Melo G, da Silva-Lima KR, Luzia-Leite J. The healing cost: comfort and discomfort experiences of women undergoing brachytherapy. Esc Anna Nery. 2016; 20(2): 317-23. https://cutt.ly/ZnGKINq

15. De Araújo-Lamino D, Turrini RNT, Kolcaba K. Cancer patients caregivers comfort. Rev. esc. enferm. USP. 2014; 48(2): 278-84. https://doi.org/10.1590/Soo80-623420140000200012

16. Rezende VL, Derchain S, Botega NJ, Sarian LO, Landulfo-Vial D, Siani-Morais S et al. Avaliação psicológica dos cuidadores de mulheres com câncer pelo. General Comfort Questionnaire. Paidéia.2010; 20(46): 229-37.

17. Medina-Jiménez N, Valenzuela-Suazo S, Orellana-Yáñez A. Impacto de un proyecto piloto de estimulación cognitiva sobre el bienestar psicoespiritual en adultos mayores. 2018; 29(2): 88-97. https://cutt.ly/inGLyh2

18. Da Rosa-dos Reis TL, Cardoso-de Paula C, Potrich T, De Mello-Padoin SM, Bin A, Flores-Mutti C, et al. Relações estabelecidas pelos profissionais de enfermagem no cuidado às crianças com doença oncológica avançada. Aquichan. 2014; 14(4): 496-508. https://doi.org/10.5294/aqui.2014.14.4.5

19. Kolcaba K. Comfort line [Internet]. Webmaster Paul Cantlay; 1997 [actualizado 2019; acceso mayo 10 de 2017]. https://bit.ly/3841C5T

20. Kolcaba K. Comfort. En: Peterson SJ, Bredow TS. Middle range theories. Application to nursing research. $3^{\text {rd }}$ ed. Philadelphia: Wolters Kluwer Health/Lippicott Williiams \& Wilkins; 2003.

21. Dowd T. La teoría del confort. En: Raile-Alligood M. Modelos y teorías en enfermería. $8^{\circ}$ ed. Barcelona, España: Elsevier; 2015.

22. Villarroel-Cruz A, Güitron-Ocaranza MP. Tratamiento del dolor en niños con cáncer. Rev. mex. anestesiol. 2015; 38(Supl. 1): 113-7. https://bit.ly/3uMfpYk

23. Cabral-Gallo MC, Delgadillo-Hernández AO, Flores-Herrera EM, et al. Manejo de la ansiedad en el paciente pediátrico oncológico y su cuidador durante la hospitalización a través de musicoterapia. Psicooncología. 2014; 11(2-3): 243-58. https://bit.ly/3sOMEIV

24. Ortigosa-Quiles JM, Riquelme-Marín A, Álvarez-Soto H, Astillero-Fuentes MJ, Sánchez-Piñera I.Eficacia de la actuación de los payasos sobre el miedo a procedimientos dolorosos en oncohematología pediátrica. Psicooncología. 2016; 13(2-3): 297-305.

25. Kolcaba KY. A theory of holistic comfort for nursing. J Adv Nurs. 1994; 19(6): 1178-84. https://doi.org/10.1111/j.1365-2648.1994.tbo1202.x

26. Superintendencia de Salud. Cáncer en personas menores de 15 años. Problema de salud 14. Santiago, Chile: Minsal; 2017. https://bit.ly/3uMg5gr

27. Ministerio de Salud. Guías Clínicas AUGE. Santiago, Chile: MINSAL; 2015. https://bit.ly/3b7oXT1

28. Kolcaba K, DiMarco MA. Comfort theory and its application to pediatric nursing. Pediatr Nurs. 2005; 31(3): 187-94. https://bit.ly/3081Rux 